\title{
Cytohistological discrepancies of cervico-vaginal smears and HPV status
}

\author{
Ibrahim Alanbay ${ }^{1}$, Mustafa Öztürk ${ }^{2}$, Fahri B. Fıratlıgil' ${ }^{3}$, \\ Kazım E. Karaşahin ${ }^{1}$, Müfit C. Yenen ${ }^{1}$, Serkan Bodur ${ }^{1}$ \\ 'Gulhane Military Medical Faculty, Department of Obstetrics and Gynecology, Etlik-Ankara, Turkey \\ 2Etimesgut Military Hospital, Department of Obstetrics and Gynecology, Etlik-Ankara, Turkey \\ ${ }^{3}$ Hakkari Military Hospital, Department of Obstetrics and Gynecology, Hakkari, Turkey
}

\begin{abstract}
Objectives: Discrepancies between abnormal cervical cytology or high-risk human papillomavirus (HR-HPV) status (cytology negative/HPV positive) and subsequent histological findings are a common occurrence. After using co-testing, the discrepancies between the HR-HPV status and cervical cytology have become an issue. In this study, we aimed to determine the characteristics of women with a discrepancy between histology and cytology/HR-HPV status, in terms of diagnosis, review and identification.

Material and methods: A total of 52 women, patients of the University Hospital between 2013-2015, with cytohistological or HR-HPV status discrepancy were recruited for the study and retrospectively analyzed. The cytological samples were liquid-based Pap smears, classified according to the 2001 Bethesda system. The HR-HPV status was identified using the Hybrid Capture 2 HR-HPV DNA assay. The histological samples were obtained by cervical biopsy as well as large loop excision of the transformation zone (LLETZ).

Results: A cytohistological discrepancy was demonstrated in patients with (-)cytology/HR-HPV(+), ASCUS, LSIL, ASC-H, HSIL, AGC-NOS: $17.3 \%, 23.07 \%, 26.9 \%, 9.5 \%, 17.3 \%$ and $5.7 \%$, respectively. When the degree of atypia in cytology increases, the concurrency of cervical cytology with biopsy also increases. A positive HR-HPV co-test result (19/24, 79.1\%) was observed in nearly all CIN2 $\geq(+)$ cases. Our study emphasizes the significance of HR-HPV testing to determine CIN2 $\geq(+)$ cases, even in the presence of a normal cytological result.

Conclusions: In case of cytohistological or HR-HPV discrepancies, a careful review of the HR-HPV status and the degree of cytological atypia should be performed before further intervention.
\end{abstract}

Key words: cervical cytology, cytology/HR-HPV discrepancy, cytological atypia

Ginekologia Polska 2017; 88, 5: 235-238

\section{INTRODUCTION}

Discrepancies between abnormal cytology and histological observations are abundant, and are also associated with significant clinical diagnostic difficulties [1]. The discrepancy rate between cytology and the subsequent histology of cervical biopsy has been estimated at $8-28 \%$ out of all smear/biopsy pairs [2-5]. The presence of discrepancies is essentially related to diagnostic inaccuracy and sampling errors, as well as non-cervical pathology sampling during colposcopy [1, 5]. A discrepancy between cervical cytology and histology can cause a diagnostic conflict, which needs careful examination before treatment, since it has been shown that inaccuracies in the histological diagnoses affect the clinical management of the discrepancies [5]. Particularly, the possibility of unrecognized disease in the cervical canal or in the lower genital tract should be taken into account when the patient is diagnosed with a negative cervical biopsy or severe cytological abnormalities [5].

The HPV test is considered to be an important testing method to confirm the cytology/histology discrepancy. Nevertheless, further evaluations need to be performed. In this study, we aimed to investigate the histopathological results 
and the HPV status of patients with a cytology/histology discrepancy.

\section{MATERIAL AND METHODS}

Between 2013 and 2015, all patients involved in the study were evaluated retrospectively for a suspected cytology/histology discrepancy. Fifty-two cases of cytology/histology discrepancies were identified from the colposcopy database, patient chart reviews, and the results of the pathology department.

All cervical smears were obtained within 2 months since the initial cytology and matched with the following endocervical curettage samples or punch biopsies. These smears constituted the referral Pap smears.

The cytology/histology discrepancy was defined as the variation between the cytological and histological diagnosis of one to two grades (major or minor) (Fig. 1), e.g. negative cytology and cervical intraepithelial neoplasia (CIN) 1 or more on biopsy, or severe cytological diagnosis and negative histological diagnosis or lower histological diagnosis on biopsy.

A discrepancy is considered to be 'major' in cases with high-grade cytological AGC (atypical glandular cell), ASC-H (atypical squamous cells cannot exclude high-grade squamous intraepithelial lesion) or HSIL (high-grade squamous intraepithelial lesion) report and histologically benign epithelium (no CIN) or CIN 1. A'minor'discrepancy is identified as a case with low-grade cytological ASCUS (atypical squamous cells of undetermined significance) or LSIL (low-grade squamous intraepithelial lesion) report and histological CIN 2-3, or cytological ASC-H, HSIL and histological CIN 2. Histological findings were characterized as follows: no lesion, CIN1, CIN2, CIN3.

All cytology samples were evaluated with liquid-based cytology and all histological samples were obtained during colposcopy-guided biopsy or large loop excisions of the transformation zone (TZ) (LLETZ). The study population were managed according to the 2012 American Society for Colposcopy and Cervical Pathology (ASCCP) guidelines [6].

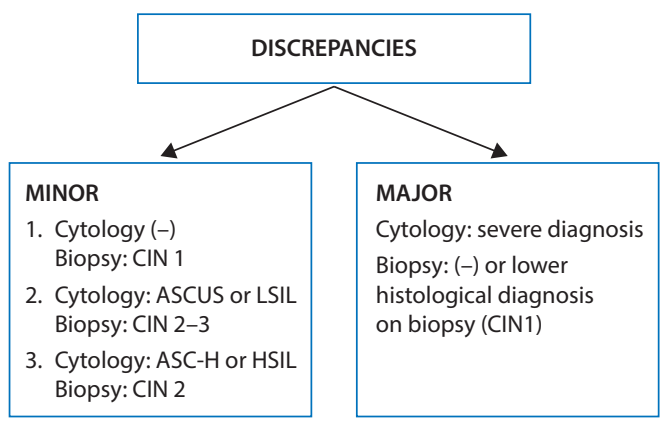

Figure 1. Classification of the discrepancies
Cytological findings were classified according to the 2001 Bethesda system. During the study period, cervical cytology samples were processed at the Department of pathology, University Hospital.

Patients with ASCUS HPV-positive were included in this study but those with ASCUS HPV-negative tests were excluded. Women with unsatisfactory cytology, cytology: NILM (negative for intraepithelial lesion or malignancy) but EC/TZ reported as absent/insufficient, and ASCUS with HPV(+) were referred to colposcopy. Subjects with ASCUS and HPV(-) were referred to co-testing in 3 years, but 2 patients insisted on colposcopy due to anxiety, and they were included in the study. Women with LSIL or higher cytology, regardless of the HPV test result, were referred to colposcopy. All detected abnormalities were biopsied. If colposcopy showed no lesion in the cervical quadrant, a random biopsy was obtained at the squamocolumnar junction in that quadrant.

\section{RESULTS}

Mean patient age was 38.2 years. The cytological results were as follows: negative, ASCUS, LSIL, HSIL, AGC-NOS: 9, 12 , 14, 5, 9, 3 (total: 52) patients, respectively. The LSIL cytology result accounted for the major group of discrepancies and the discrepancy rates were (\%) 26.9, 23.7, 17.3, 17.3, 9.6, 5.7 for LSIL, ASCUS, negative cytology, HSIL, ASC-H and AGC-NOS, respectively. The rate of histologically benign epithelium (no CIN) was 6 (50\%) in ASCUS, 5 (39.2\%) LSIL, 2 (40\%) AC-H, 4 (44.4\%) HSIL, and 1 (33.3\%) AGC-NOS cytology, respectively (percentages are within each subgroup). Eighteen subjects (34.6\%) were found to be $\geq$ ASCUS, but the histology was negative and inflammatory (minor discrepancy). In 8 (15.3\%) cases, the referral cytology was high-grade (ASC-H and HSIL), but the histology was low-grade (<CIN 3; minor discrepancy).

In the major discrepancy group, out of 22 (42.3\%) cases, the negative referral cytology report was 5, ASCUS - 6, LSIL -9 , and AGC-NOS - 2 cases, but their histology samples were reported as $\geq$ CIN2. Out of 9 (17.3\%) patients, the referral cytology was negative, but the histology was CIN 1 for 6 cases (minor discrepancy), and CIN 3 for 3 cases (major discrepancy).

In summary, total major discrepancy rate was 24 (46\%). The major discrepancy rate was higher in the HSIL group, $77.7 \%(7 / 9)$, and ASC-H group 60\% (3/5) as compared to cytology (-) 55.5\% (5/9), LSIL 50\% (6/12), and ASCUS 21.4\% (3/14). As a result, there were 10 (19.2\%) colposcopy-confirmed CIN 3 patients with missed diagnosis of high-grade $\mathrm{CIN}$ in cytology. The details of cytohistological discrepancies are presented in Table 1.

The HR-HPV positive rate was 79.1\% (19/24) in colposcopy-confirmed CIN 2-3 groups, while this rate was $11.5 \%$ (3/26) in benign and CIN 1 groups. The HR-HPV positive rate 
Table 1. Referral cytology and cervical biopsy diagnosis for women referred with a cytology/histology discrepancy ( $\mathrm{n}=52$ )

\begin{tabular}{|l|c|c|c|c|c|c|c|c|c|c|}
\hline & \multicolumn{9}{|c|}{ Histopathologic diagnosis/HPV (n) } \\
\hline Cytological diagnosis $(\mathbf{n})$ & No CIN & HPV & CIN 1 & HPV & CIN2 & HPV & CIN3 & HPV & Total \% \\
\hline Negative (9) & - & & 4 & & 2 & & 3 & 3 & 17.3 \\
\hline ASCUS (12) & 6 & -5 & - & & 4 & 2 & 2 & 2 & 23.07 \\
\hline LSIL (14) & 5 & 1 & - & & 6 & 3 & 3 & 3 & 26.9 \\
\hline ASC-H (5) & 2 & - & 1 & 1 & 2 & 2 & - & - & 09.6 \\
\hline HSIL (9) & 4 & - & 3 & 1 & 2 & 1 & - & - & 17.3 \\
\hline AGC-NOS (3) & 1 & - & - & & - & - & 2 & - & 5.7 \\
\hline Total (52) (\%) & 34.6 & & 15.3 & & 30.7 & & 19.2 & & \\
\hline
\end{tabular}

*The ASCUS group included HPV(+) ASCUS and HPV(-) ASCUS but referred to colposcopy due to anxiety (3 cases)

was $100 \%$ in subjects with referral cytology (-), ASCUS, and LSIL, but histology CIN 3. HPV positivity was associated with an increase in the major discrepancy rates among cytohistological discrepancies (Tab. 1).

\section{DISCUSSION}

According to the literature, the discrepancy rate between the referral smear and the subsequent histology ranges between $8 \%$ and $28 \%$ [2-5]. However, there is significant heterogeneity between the type of cervical biopsy and identification of the study population in the background population [5, 7]. Cytological errors are considered to be the main reasons of cytohistological discrepancies. The reason of these cytological errors lies not only in cytological sampling errors, but also suboptimal staining quality, poor specimen preservation, as well as cytological interpretation errors [5].

A study of 670 smears coupled with subsequent cervical punch biopsy or curettage which were performed within 2 months, found 60 cases (9\%) diagnosed as cytohistological discrepancy. For the cases present in the sampling error group, errors related to cytology sampling were detected in 40 subjects, while in 11 cases the errors were due to biopsy sampling [8]. The size and type of the cervical biopsy, as well as inadequate and inaccurate sampling of the transformation zone, have been determined as the most common causes of the discrepancy [7].

The concurrence of cervical cytology with biopsy discrepancy increases with the degree of atypia in cytology. Another study showed that nearly $100 \%$ of cytohistological discrepancy cases were reported for $\mathrm{HSIL}$, while this rate was reduced to $54 \%$ for ASCUS and $20 \%$ for AGC [9]. Heatley et al., showed that the correlation between punch biopsy and the highest grade of CIN was only 63\% [10]. In our study, LSIL cytology results were found to be the major group of discrepancy. The discrepancy rates were 26.9\%, 23.7\%, 17.3\%, $17.3 \%, 9.6 \%$, and $5.7 \%$ for LSIL, ASCUS, negative cytology, HSIL, ASC-H and AGC-NOS, respectively.
Cytohistological discrepancy among women with HSIL in histology is a matter of critical importance because less aggressive management would result in delayed diagnosis and treatment [11]. In our study, there were 10 (19.2\%) colposcopy-confirmed CIN3 patients with missed diagnosis of high grade CIN in cytology. Importantly, accurate colposcopic diagnosis depends on many factors, i.e. colposcopic findings, including the size of lesion, whether the colposcopy was adequate, and the experience of the colposcopist. Considerable variations in accurate diagnosis of colposcopy-directed biopsy have been reported by various authors $[12,13]$. The ability of the colposcopy-directed biopsy to accurately detect the severity of cervical abnormality has been a major question in several studies, with the false-negative rate of $50 \%$. In one study, the rate of unnoticed $\geq$ CIN2(+) disease in women with minor cytological abnormalities and minor colposcopic changes was 31\% [11].

The relation between persistent infections caused by human papillomavirus (HPV) and the development of cervical cancer has been demonstrated [14]. The prevalence of HPV has been reported as 70-90\% in CIN 2-3 [15]. A study of 252 high-grade cervical neoplasia cases showed that $98 \%$ of them were HR-HPV positive [14]. A recently developed clinical trial, Addressing the Need for Advanced HPV Diagnostics (ATHENA), demonstrated that HR-HPV (14 types) was observed in $65 \%$ of the women with CIN1, $83.3 \%$ with CIN2, and $92.6 \%$ with CIN3 [16], which is consistent with our study: $\mathrm{HPV}(+)$ rate $-79.1 \%(19 / 24)$ in CIN2 and CIN3 groups which were colposcopically confirmed, and $11.5 \%$ in CIN1 and no CIN groups.

The utility of the Papanicolaou test has been increasingly questioned as several meta-analyses have found the test inaccurate in detecting cervical cancers. It failed to diagnose about $50 \%$ of clinically significant pre-cancers and cancers which were present at the time of observation [17]. Thus, recently, the high-risk HPV (HR-HPV) test has become more reliable to detect and manage cervical squamous cancer, CIN2 and CIN3. 
The strength of this study lies in our using cervical biopsy specimens to confirm the final histological diagnosis. Regardless, our study was not without limitations, namely lack of data about sexual behavior and history of previous Pap testing, which might be related to cytohistological discrepancy.

\section{CONCLUSIONS}

As was demonstrated in our study, the relation of HR-HPV with the major discrepancies between cytology and histopathology is in accordance with the 2013 ASCCP guidelines, which suggested that patients with discrepancies between HR-HPV and cytology reports should undergo colposcopy and biopsy [6]. According to the recent colposcopic principles, the sensitivity in detecting high-grade CIN should be increased using cytology-based screening in the presence of high-grade referral cytology or HPV positivity [6].

\section{Conflicts of interests}

Authors declare that there are no conflicts of interest.

\section{REFERENCES}

1. Skaggs V, Moore K, Stowell S, et al. A LEEP cervical conization is rarely indicated for a two-step discrepancy. J Low Genit Tract Dis. 2007; 11(3): 134-137, doi: 10.1097/01.Igt.0000265776.29173.e1, indexed in Pubmed: 17596756.

2. $\mathrm{ChoH}, \mathrm{Kim} \mathrm{JH}$. Treatment of the patients with abnormal cervical cytology: a. J Gynecol Oncol. 2009; 20(3): 164-168, doi:10.3802/jgo.2009.20.3.164, indexed in Pubmed: 19809550.

3. Li ZG, Qian deY, Cen JM, et al. Three-step versus "see-and-treat" approach in women with high-grade squamous intraepithelial lesions in a low-resource country. Int J Gynaecol Obstet, 2009;106: 202. 2009; 106(3): 202-205, doi: 10.1016/j.ijgo.2009.04.011.

4. Kirby TO, Leath CA. 3rd, Huh WK, et al. A prospective evaluation of "see and treat" in women with HSIL Pap smear results: is this an appropriate strategy? J Low Genit Tract Dis. 2005; 9(2): 6.

5. Moss EL, Moran A, Douce G, et al. Cervical cytology/histology discrepancy: a 4-year review of patient outcome. Cytopathology. 2010; 21 (6): 389-394, doi: 10.1111/j.1365-2303.2010.00754.x, indexed in Pubmed: 20482721.
6. Massad LS, Einstein MH, Huh WK, et al. 2012 ASCCP Consensus Guidelines Conference. 2012 updated consensus guidelines for the management of abnormal cervical cancer screening tests and cancer precursors. Obstet Gynecol. 2013; 121(4): 829-846, doi:10.1097/AOG.0b013e3182883a34, indexed in Pubmed: 23635684.

7. Tritz DM, Weeks JA, Spires SE, et al. Etiologies for non-correlating cervical cytologies and biopsies. Am J Clin Pathol. 1995; 103(5): 594-597, doi: 10.1093/ajcp/103.5.594, indexed in Pubmed: 7741105.

8. Izadi-Mood N, Sarmadi S, Sanii S. Quality control in cervicovaginal cytology by cytohistological correlation. Cytopathology. 2013; 24(1): 33-38, doi: 10.1111/j.1365-2303.2011.00926.x, indexed in Pubmed: 21929578.

9. Turkmen I, Bassullu N, Korkmaz P, et al. Patients with epithelial cell abnormality in pap smears: correlation of results with follow-up smears andcervical biopsies. Turkish Journal of Pathology. 2013, doi: 10.5146/tjpath.2013.01182.

10. Heatley MK, Bury JP. The correlation between the grade of dyskaryosis on cervical smear, grade of cervical intraepithelial neoplasia (CIN) on punch biopsy and the final histological diagnosis on cone biopsies of the cervix. Cytopathology. 1998; 9(2): 93-99, indexed in Pubmed: 9577735.

11. Moss EL, Hadden P, Douce G, et al. Is the colposcopically directed punch biopsy a reliable diagnostic test in women with minor cytological lesions? J Low Genit Tract Dis. 2012; 16(4): 421-426, doi: 10.1097/LGT.0b013e318250acf3, indexed in Pubmed: 22669079.

12. Baldauf JJ, Dreyfus M, Ritter J, et al. An analysis of the factors involved in the diagnostic accuracy of colposcopically directed biopsy. Acta Obstetricia et Gynecologica Scandinavica. 1997; 76(5): 468-473, doi: 10.3109/00016349709047830.

13. Chappatte OA, Byrne DL, Raju KS, et al. Histological differences between colposcopic-directed biopsy and loop excision of the transformation zone (LETZ): a cause for concern. Gynecol Oncol. 1991; 43(1): 46-50, indexed in Pubmed: 1959787.

14. Marel Jv, Berkhof J, Ordi J, et al. Attributing Oncogenic Human Papillomavirus Genotypes to High-grade Cervical Neoplasia. The American Journal of Surgical Pathology. 2015; 39(4): 496-504, doi: 10.1097/PAS.0000000000000342.

15. Bosch FX, Manos MM, Muñoz N, et al. Prevalence of human papillomavirus in cervical cancer: a worldwide perspective. International biological study on cervical cancer (IBSCC) Study Group. J Natl Cancer Inst. 1995; 87(11): 796-802, doi: 10.1093/jnci/87.11.796, indexed in Pubmed: 7791229.

16. Wright TC, Stoler MH, Behrens CM, et al. The ATHENA human papillomavirus study: design, methods, and baseline results. Am J Obstet Gynecol. 2012; 206(1): 46.e1-46.e11, doi: 10.1016/j.ajog.2011.07.024, indexed in Pubmed: 21944226

17. Louvanto K, Chevarie-Davis M, Ramanakumar AV, et al. HPV testing with cytology triage for cervical cancer screening in routine practice. Am J Obstet Gynecol. 2014; 210(5): 474.e1-474.e7, doi: 10.1016/j. ajog.2013.12.033, indexed in Pubmed: 24373948. 